\title{
The Analysis of Noise Frequency Modulation Jamming Signal Based on Stochastic Differential
}

\author{
Jingbo He \\ electronics eng.college, naval univ. of engineering, \\ wuhan 430033, china;
}

\author{
Fuyuan Peng \\ department of electronics and information engineering, \\ huazhong university of science and technology \\ wuhan 430074, china
}

\author{
Zhong Liu \\ electronics eng.college, naval univ. of engineering \\ wuhan 430033, china;
}

\begin{abstract}
It is the most important problem in the radar countermeasures that the radar jamming effectiveness evaluation. The basis of the radar jamming effectiveness evaluation is the radar jamming signal processing. According to the intrinsic relations between the stochastic differential and the radar jamming signal processing, the stochastic calculus was used in the radar jamming signal processing in this paper. The noise frequency modulation signal was particularly analyzed. The Fokker-Planck equation of noise frequency modulation was presented and the Motion-Group Fourier Transform was used by converting the partial differential equation into the variable coefficient homogenous linear differential equations. Then the solutions were given by using the peano-baker series.
\end{abstract}

Keywords-Stochastic Differential, Fokker-Planck Equation, Motion-Group Fourier Transform (MGFT), Peano-Baker series

\section{INTRODUCTION}

It is the most important problem in the radar countermeasures that the radar jamming effectiveness evaluation. The basis of the radar jamming effectiveness evaluation is the radar jamming signal processing. That is how to get the probability density function (PDF) with the jamming signal. It is so difficult that many models are in the basis of statistic method which only described the relationship between PDF and signal noise ratio (SNR) in [1] [5]. But PDF is connection with multi-parameters including noise variance, frequency of carrier wave and amplitude of carrier wave.

A new method that describe the relationship between phase noise (PN) and the filtered signal is found in [6] [9]. According to this, we presented the models that describe the relationship between noise frequency modulation and pulse compression radar signal. The Fokker-Planck (FP) equation of noise frequency modulation is presented and the MotionGroup Fourier Transform (MGFT) is used by converting the partial differential equation into the variable coefficient homogenous linear differential equations. Also peano-baker series are used to solve the variable coefficient homogenous linear differential equations.

\section{THE IMPACT OF NOISE FREQUENCY} MODULATION JAMMING SIGNAL

\section{A. The Statistical Character of Noise Frequency}

\section{Modulation Jamming Signal}

The noise frequency modulation jamming signal can be written as

$$
J(t)=U_{j} \cos \left[2 \pi f_{j} t+2 \pi K_{F M} \int_{0}^{t} u(s) d s\right]
$$

where $U_{j}$ is the amplitude of carrier wave, $f_{j}$ is the frequency of jamming carrier wave, $K_{F M}$ is the frequency modulation coefficient. $u(s)$ is the white noise with zero mean and variance of $\sigma_{n}^{2}$. The correlation function of $J(t)$ is

$$
B_{j}(\tau)=\frac{U_{j}^{2}}{2} e^{-\frac{\sigma^{2}(\tau)}{2}} \cos 2 \pi f_{j} \tau
$$

where $e(t)=\int_{0}^{t} u(s) d s$ is coincidence with $N\left(0, \sigma_{n}^{2} t\right)$,

$$
\sigma^{2}(\tau) \text { is the variance of frequency modulation }
$$

$$
\begin{aligned}
2 & \pi K_{F M}[e(t+\tau)-e(t)] . \text { Then } \\
\sigma^{2}(\tau) & =4 \pi^{2} K_{F M}^{2} E[e(t+\tau)-e(t)]^{2} \\
& =4 \pi^{2} K_{F M}^{2} E\left[e^{2}(t+\tau)-2 e(t+\tau) e(t)+e^{2}(t)\right] \\
& =4 \pi^{2} K_{F M}^{2} \sigma_{n}^{2}[(t+\tau)-2 t+t] \\
& =4 \pi^{2} K_{F M}^{2} \sigma_{n}^{2} \tau
\end{aligned}
$$

From (2) and (3), we can get

$$
B_{j}(\tau)=\frac{U_{j}^{2}}{2} e^{-2 \pi^{2} K_{F M}^{2} \sigma_{n}^{2} \tau} \cos 2 \pi f_{j} \tau
$$

Obviously correlation function $B_{j}(\tau)$ is only relation with time difference $\tau$ and satisfies the condition of broad steady. In the following, we will derive the stochastic differential 
equations (SDEs) first. Then we write the corresponding FP equations. At last we use the MGFT to solve the FP equations.

\section{B. The Fokker-Planck Equation}

The input noise frequency modulation jamming signal of radar receiver intermediate frequency (IF) filter is $s(t)=U_{j} e^{j\left(2 \pi f_{j} t+2 \pi K_{F M} \int_{0}^{t} u(s) d s\right)}=U_{j} e^{j \phi(t)} \cdot h(t)$ is the impulse response of the matched IF filter. The output is

$$
z(t)=h(t) * s(t)=\int_{0}^{t} h(\tau) U_{j} e^{j[\phi(t)-\phi(\tau)]} d \tau
$$

Let write $\phi_{\Delta t}=\phi(t+\Delta t)-\phi(t)$, then

$$
\begin{aligned}
z(t+\Delta t) & =\int_{0}^{t+\Delta t} h(\tau) U_{j} e^{j[\phi(t+\Delta t)-\phi(\tau)]} d \tau \\
& =\int_{0}^{t} h(\tau) U_{j} e^{j[\phi(t)-\phi(\tau)]} e^{j \phi_{\Delta t}} d \tau \\
& +\int_{t}^{t+\Delta t} h(\tau) U_{j} e^{j[\phi(t+\Delta t)-\phi(\tau)]} d \tau \\
& =e^{j \phi_{\Delta t}} \int_{0}^{t} h(\tau) U_{j} e^{j[\phi(t)-\phi(\tau)]} d \tau \\
& +\int_{t}^{t+\Delta t} h(\tau) U_{j} e^{j[\phi(t+\Delta t)-\phi(\tau)]} d \tau \\
& =z(t) e^{j \phi_{\Delta t}}+\int_{t}^{t+\Delta t} h(\tau) U_{j} e^{j[\phi(t+\Delta t)-\phi(\tau)]} d \tau
\end{aligned}
$$

At the time interval $t \leq \tau \leq t+\Delta t$,

$$
\begin{aligned}
& \phi(t+\Delta t)-\phi(\tau) \sim N\left(2 \pi f_{j}(t+\Delta t-\tau),\right. \\
& \left.4 \pi^{2} K_{F M}^{2} \sigma_{n}^{2}(t+\Delta t-\tau)\right)
\end{aligned}
$$

and $\phi(t+\Delta t)-\phi(\tau)=o\left(2 \pi f_{j} \Delta t+2 \pi K_{F M} \sigma_{n} \sqrt{\Delta t}\right)$. The integral in (6) is

$$
\begin{aligned}
& \int_{t}^{t+\Delta t} h(\tau) U_{j} e^{j o\left(2 \pi f_{j} \Delta t+2 \pi K_{F M} \sigma_{n} \sqrt{\Delta t}\right)} d \tau \\
& =U_{j} h(t) e^{j o\left(2 \pi f_{j} \Delta t+2 \pi K_{F M} \sigma_{n} \sqrt{\Delta t}\right)} \Delta t \\
& =U_{j} h(t)\left[1+j o\left(2 \pi f_{j} \Delta t+2 \pi K_{F M} \sigma_{n} \sqrt{\Delta t}\right)\right] \Delta t \\
& =U_{j} h(t)[\Delta t+o(\Delta t)]
\end{aligned}
$$

Substitute (8) into (6), we have

$$
z(t+\Delta t)=z(t) e^{j \phi_{\Delta t}}+U_{j} h(t)[\Delta t+o(\Delta t)]
$$

So

$$
\begin{aligned}
d z(t) & =U_{j} h(t) d t+j z(t)\left[2 \pi f_{j} d t+2 \pi K_{F M} \sigma_{n} d W(t)\right] \\
& =\left[U_{j} h(t)+j 2 \pi f_{j} z(t)\right] d t+j 2 \pi K_{F M} \sigma_{n} z(t) d W(t)
\end{aligned}
$$

Let $z(t)=r(t) e^{j \theta(t)}$, we rewrite (10) in polar coordinates as

$$
\begin{aligned}
& d r(t) e^{j \theta(t)}=\left[U_{j} h(t)+j 2 \pi f_{j} r(t) e^{j \theta(t)}\right] d t \\
& +j 2 \pi K_{F M} \sigma_{n} r(t) e^{j \theta(t)} d W(t)
\end{aligned}
$$

The left of (11) is

$$
\begin{aligned}
& e^{j \theta(t)} d r(t)+j r(t) e^{j \theta(t)} d \theta(t) \\
& =[\cos \theta(t) d r(t)-r(t) \sin \theta(t) d \theta(t)] \\
& +j[\sin \theta(t) d r(t)+r(t) \cos \theta(t) d \theta(t)]
\end{aligned}
$$

and the right is

$\left[U_{j} h(t)+j 2 \pi f_{j} r(t) e^{j \theta(t)}\right] d t+j 2 \pi K_{F M} \sigma_{n} r(t) e^{j \theta(t)} d W(t)$

$=\left[U_{j} U_{0} B \cos \left(2 \pi f_{0} t-\pi \frac{B}{T} t^{2}\right) d t-2 \pi f_{j} r(t) \sin \theta(t) d t\right.$

$\left.-2 \pi K_{F M} \sigma_{n} r(t) \sin \theta(t) d W(t)\right]$

$+j\left[U_{j} U_{0} B \sin \left(2 \pi f_{0} t-\pi \frac{B}{T} t^{2}\right) d t+2 \pi f_{j} r(t) \cos \theta(t) d t\right.$

$\left.+2 \pi K_{F M} \sigma_{n} r(t) \cos \theta(t) d W(t)\right]$

From (11) to (13), we can get

$$
\begin{aligned}
& {\left[\begin{array}{l}
d r(t) \\
d \theta(t)
\end{array}\right]=\left[\begin{array}{c}
U_{j} U_{0} B[\cos \varphi(t) \cos \theta(t)+\sin \varphi(t) \sin \theta(t)] \\
2 \pi f_{j}+\frac{U_{j} U_{0} B[\sin \varphi(t) \cos \theta(t)-\cos \varphi(t) \sin \theta(t)]}{r(t)}
\end{array}\right] d t} \\
& +\left[\begin{array}{c}
0 \\
2 \pi K_{F M} \sigma_{n}
\end{array}\right] d W(t)
\end{aligned}
$$

where $\varphi(t)=2 \pi f_{0} t-\pi \frac{B}{T} t^{2}$. we can derive the FokkerPlanck equation for (14)as:

$$
\begin{aligned}
\frac{\partial p}{\partial t} & =-\sum_{i=1}^{2} \frac{\partial}{\partial x_{i}}\left[a_{i} p(x, t)\right]+\frac{1}{2} \sum_{i, j=1}^{2} \frac{\partial^{2}}{\partial x_{i} \partial x_{j}}\left[\left(H H^{T}\right)_{i j} p(x, t)\right] \\
& =-U_{j} U_{0} B[\cos \varphi(t) \cos \theta(t)+\sin \varphi(t) \sin \theta(t)] \frac{\partial p}{\partial r} \\
& -\left[2 \pi f_{j}+\frac{U_{j} U_{0} B[\sin \varphi(t) \cos \theta(t)-\cos \varphi(t) \sin \theta(t)]}{r(t)}\right] \frac{\partial p}{\partial \theta} \\
& +\frac{\left(2 \pi K_{F M} \sigma_{n}\right)^{2}}{2} \frac{\partial^{2} p}{\partial \theta^{2}}
\end{aligned}
$$

C. Solving the Fokker-Planck Equation Using MGFT

Applying the MGFT to (15), we can convert it to an infinite system of linear ordinary differential equations (ODEs) 


$$
\begin{aligned}
\frac{d \hat{p}}{d t} & =-U_{j} U_{0} B \cos \varphi(t) \hat{p} \eta\left(\tilde{X}_{2}, p\right)-U_{j} U_{0} B \sin \varphi(t) \hat{p} \eta\left(\tilde{X}_{3}, p\right) \\
& +2 \pi f_{j} \eta\left(\tilde{X}_{1}, p\right) \hat{p}-2 \pi f_{j} \hat{p} \eta\left(\tilde{X}_{1}, p\right)+\frac{\left(2 \pi K_{F M} \sigma_{n}\right)^{2}}{2}\left[\eta\left(\tilde{X}_{1}, p\right)\right]^{2} \hat{p} \\
& +\frac{\left(2 \pi K_{F M} \sigma_{n}\right)^{2}}{2} \hat{p}\left[\eta\left(\tilde{X}_{1}, p\right)\right]^{2}-\left(2 \pi K_{F M} \sigma_{n}\right)^{2} \eta\left(\tilde{X}_{1}, p\right) \hat{p} \eta\left(\tilde{X}_{1}, p\right)
\end{aligned}
$$

Once we get the solution to the ODE (16), we can then substitute it into the Fourier inversion formula for the motion group to recover the PDF $p(r, \theta, \phi ; t)$. To get the joint $\mathrm{PDF} p(r, \theta ; t)$ is just an integration, with respect to $\phi$, as

$$
\begin{aligned}
p(r, \theta ; t) & =\frac{1}{2 \pi} \int_{0}^{2 \pi} p(r, \theta, \phi ; t) d \phi \\
& =\sum_{n \in Z} j^{-n} e^{-j n \theta} \int_{0}^{\infty} \hat{p}_{0, n}(p) J_{-n}(p r) p d p
\end{aligned}
$$

Integrating (17) over $\theta$ will give us the marginal PDF of $z(t)$ as

$$
p(r ; t)=\int_{0}^{\infty} \hat{p}_{0,0}(p) J_{0}(p r) p d p
$$

\section{Solving the Variable Coefficients Homogenous Linear} Differential Equations

(16) is the variable coefficients homogenous linear differential equations. We use peano-baker series to solve it. The algorithm is as follows:

1) Dividing time domain $[0, t]$ into $N$ intervals and Using peano-baker series to calculate the state transfer function at any time interval $\left[t_{j}, t_{j+1}\right]$ $\Phi\left(t_{j}, t_{j+1}\right)=I+\left[A\left(t_{j}\right)+A\left(t_{j+1}\right)\right] \tau / 2+A^{2}\left(t_{j}\right) \tau^{2} / 2$

where $\tau=t / N, A$ is the coefficients matrix of variable coefficients homogenous linear differential equations.

2) Depending on the quality of state transfer function, the whole state transfer function is

$$
\Phi(0, t)=\Phi\left(t_{N-1}, t_{N}\right) \Phi\left(t_{N-2}, t_{N-1}\right) \cdots \Phi\left(t_{0}, t_{1}\right)
$$

3) The formula

$$
\hat{p}(t)=\Phi(0, t) \hat{p}(0)
$$

can be used to get the solution of variable coefficients homogenous linear differential equations.

\section{NUMERICAL RESULTS}

Setting the experiment parameters $U_{j}=1, U_{0}=1$, $T=1 \times 10^{-4}, f_{0}=1 \times 10^{5}, f_{j}=f_{0}, B=1 \times 10^{6}$ and defining the information entropy $H=-\int_{0}^{\infty} p(r) \ln p(r) d r$. The relationship between the carrier wave frequency $f_{j}$ of noise frequency modulation jamming signal and the probability of detection information entropy $H$ is described in Fig. 1.

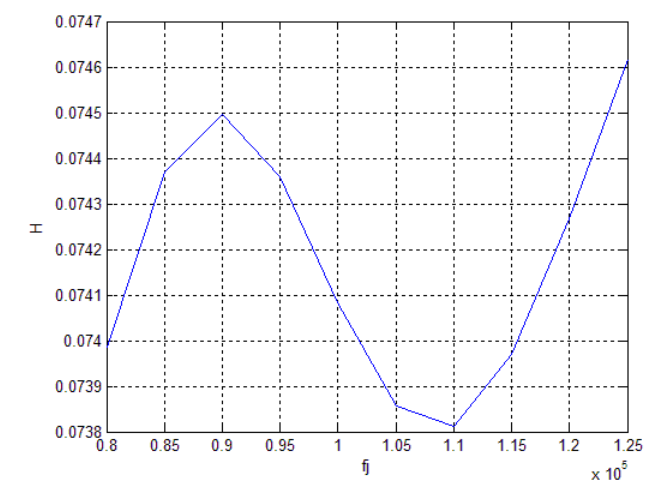

Figure 1. Probability of detection information entropy $H$ for different jamming frequency $f_{j}$

\section{SUMMARIES}

We have discussed the application of stochastic differential theory in the domain of radar jamming signal processing. We have presented the Fokker-Planck equation for the noise frequency modulation jamming signal. And the MGFT has been used by converting the partial differential equation into the variable coefficient homogenous linear differential equations. Then the solutions are given by using the peano-baker series. So the probability density function of noise frequency modulation in the filter is given. The impact of noise frequency modulation jamming signal on the pulse compression radar is analyzed in the criterion of information entropy.

\section{ACKNOWLEDGEMENTS}

This paper is supported by the National Science Foundation Project (60872076), the China Postdoctoral Science Foundation (20100471795) and Natural Science Foundation of Hubei Province of China (2009CDB337).

\section{REFERENCES}

[1] B M Welsh; J N Link. Accuracy Criteria for Radar Cross Section Measurements of Targets Consisting of Multiple Independent Scatters. IEEE TRANSACTIONS ON ANTENNAS AND PROPAGATION [J], 1988.11, PP: 1587-1593

[2] V Anastassopoulos; G A Lampropoulos; A Drosopoulos. High Resolution Radar Clutter Statistics. IEEE TRANSACTIONS ON AEROSPACE AND ELECTRONIC SYSTEMS [J], 1999.1, PP: 4360

[3] A J Rainal. Monopulse Radars Excited by Gaussian Signals. IEEE TRANSACTIONS ON AEROSPACE AND ELECTRONIC SYSTEMS [J], 1966.3, PP: 337-345

[4] $M$ Shor. Performance of Order Statistics CFAR. IEEE TRANSACTIONS ON AEROSPACE AND ELECTRONIC SYSTEMS [J], 1991.2, PP: 214-224 
[5] I R Joughin; D P Winebrenner; D B Percival. Probability Density Functions for Multilook Polarimetric Signatures. IEEE TRANSACTIONS ON GEOSCIENCE AND REMOTE SENSING [J], 1994.5, PP: 562-574

[6] G S Chirikjian; A B Kyatkin. Engineering Applications of Noncommutative Harmonic Analysis [M]. Boca Raton, FL: CRC Press, 2000

[7] W Miller. Lie Theory and Special Functions [M]. New York: Academic, 1968
[8] Y F Wang. Applications of diffusion processes in robotics, optical communications and polymer science [D]. Ph.D. dissertation, Johns Hopkins Univ., Baltimore, MD, 2001

[9] Y F Wang; Y Zhou; D K Masten. Solving Phase-Noise FokkerPlanck Equations Using the Motion-Group Fourier Transform. IEEE TRANSACTIONS ON COMMUNICATIONS [J], 2006.5, PP: 868877 